\title{
Music and Lyrics: Musical Training for Aural Rehabilitation
}

\author{
Yehree Kim (iD · Woo Seok Kang (i) \\ Department of Otorhinolaryngology-Head and Neck Surgery, Asan Medical Center, University of Ulsan College of Medicine, Seoul, Korea
}

Both music and language are unique and essential aspects of human culture that require the recognition, interpretation, and handling of complex sounds that are hierarchically arranged; in the order of syllables, words, and sentences in speech and notes, beats, and phrases in music. To process music and language, the brain must decode these signals by extracting relevant information from an ever rapidly changing sound environment. This process is cognitively demanding, and the neural circuits responsible for both music and language processing are known to be similar [1].

It has been speculated that the cognitive abilities of music and language processing transfer from one to the other through the reorganization of common neural circuits [2]. Many studies have validated this hypothesis by showing that musical training improves speech processing at the levels of perception of speech prosody, consonant contrasts, speech segmentation, and syntactic processing [3]. Individuals with vocational musical experience also tend to have better verbal memory [4]. These findings extend to the subcortical level, showing an enhancement of the neural representations of the pitch, timbre, and timing of speech sounds after musical practice that correlates with a better ability to perceive speech in noise. Overall, the advantages induced by musical training enhance the neural coding of sounds, in both cortical and subcortical structures, extending to speech sounds [1].

These benefits of musical training among normal-hearing adults have naturally led to the utilization of musical training for hearing aid and cochlear implant users. The existing literature suggests that musical training can improve perceptions, localization, differentiation, and recognition of sound. Significant improvements in appreciation of timbre and identification of melodic contours have been observed in cochlear implant users. Better tone recognition and speech perception have also been recognized in speakers of tonal languages $[5,6]$.

These previous studies do suggest a promising role of musical training in patients using various hearing devices; however, there remains much more to be explored. Many reported studies lack a well-established control group receiving a non-musical inter- vention. Related factors such as patient age, the type and mode of hearing device, and what kind of a musical program would positively impact hearing aid and/or cochlear implant users are still unclear. The minimal training period required to see a benefit has not yet been established. This duration is another important factor that would influence patient compliance and motivation. The appropriate degree and variety of the given auditory stimuli should also be investigated, and the reception of feedback that this training method is valid is also warranted.

Nevertheless, musical training is a pleasurable approach for aural rehabilitation that can enhance speech and music perception abilities, and further investigations would provide insights into individual-based rehabilitation methods.

\section{CONFLICT OF INTEREST}

No potential conflict of interest relevant to this article was reported.

\section{ORCID}

$\begin{array}{ll}\text { Yehree Kim } & \text { https://orcid.org/0000-0002-3056-198X } \\ \text { Woo Seok Kang } & \text { https://orcid.org/0000-0003-1692-4155 }\end{array}$

\section{AUTHOR CONTRIBUTIONS}

Conceptualization: WSK. Data curation, Formal analysis, Methodology, Writing-original draft, review, \& editing: all authors.

\section{REFERENCES}

1. Intartaglia B, White-Schwoch T, Kraus N, Schon D. Music training

Copyright (C) 2021 by Korean Society of Otorhinolaryngology-Head and Neck Surgery.

This is an open-access article distributed under the terms of the Creative Commons Attribution Non-Commercial License (https://creativecommons.org/licenses/by-nc/4.0)

which permits unrestricted non-commercial use, distribution, and reproduction in any medium, provided the original work is properly cited. 
enhances the automatic neural processing of foreign speech sounds. Sci Rep. 2017 Oct;7(1):12631.

2. Patel AD. Why would musical training benefit the neural encoding of speech?The OPERA hypothesis. Front Psychol. 2011 Jun;2:142.

3. Francois C, Schon D. Musical expertise boosts implicit learning of both musical and linguistic structures. Cereb Cortex. 2011 Oct;21(10): 2357-65.

4. Chan AS, HoYC, Cheung MC. Music training improves verbal memory. Nature. 1998 Nov;396(6707):128.

5. Jiam NT, Deroche ML, Jiradejvong P, Limb CJ. A randomized controlled crossover study of the impact of online music training on pitch and timbre perception in cochlear implant users. J Assoc Res Otolaryngol. 2019 Jun;20(3):247-62.

6. Shukor NF, Lee J, Seo YJ, Han W. Efficacy of music training in hearing aid and cochlear implant users: a systematic review and metaanalysis. Clin Exp Otorhinolaryngol. 2021 Feb;14(1):15-28.

Received January 11, 2021 Accepted January 12, 2021 